\title{
PALOMAR 12 AS A PART OF THE SAGITTARIUS STREAM: THE EVIDENCE FROM ABUNDANCE RATIOS ${ }^{1}$
}

\author{
Judith G. CoHeN ${ }^{2}$ \\ Received 2003 November 7; accepted 2003 December 11
}

\begin{abstract}
We present a detailed abundance analysis for 21 elements based on high-dispersion, high spectral resolution Keck spectra for four members of the outer halo "young" Galactic globular cluster Palomar 12. All four stars show identical abundance distributions with no credible indication of any star-to-star scatter. However, the abundance ratios of the Pal 12 stars are very peculiar. There is no detected enhancement of the $\alpha$-elements; the mean of $[\mathrm{Si} / \mathrm{Fe}],[\mathrm{Ca} / \mathrm{Fe}]$, and $[\mathrm{Ti} / \mathrm{Fe}]$ is $-0.07 \pm 0.05 \mathrm{dex}, \mathrm{O} / \mathrm{Fe}$ is also solar, while $\mathrm{Na}$ is very deficient. The distribution among the heavy elements shows anomalies as well. These are inconsistent with those of almost all Galactic globular clusters or of field stars in the Galaxy. The peculiarities shown by the Pal 12 stars are, however, in good general agreement with the trends established by Smecker-Hane \& McWilliam and by Bonifacio et al. for stars in the Sagittarius dwarf spheroidal (dSph) galaxy evaluated at the $[\mathrm{Fe} / \mathrm{H}]$ of $\mathrm{Pal} 12$. This reinforces earlier suggestions that Pal 12 originally was a cluster in the Sgr dSph galaxy that during the process of accretion of this galaxy by our own was tidally stripped from the Sgr galaxy to become part of the extended Sgr stream.
\end{abstract}

Key words: globular clusters: general — globular clusters: individual (Palomar 12) galaxies: individual (Sagittarius dSph) — stars: abundances

On-line material: machine-readable table

\section{INTRODUCTION}

Pal 12 is a sparse globular cluster (GC) located in the outer halo of the Milky Way. The color-magnitude diagram (CMD) studies of Gratton \& Ortolani (1988) and of Stetson et al. (1989) suggested that Pal 12 is probably somewhat younger than the vast majority of Galactic GCs. However, until there was a measurement of the metallicity of this $\mathrm{GC}$, its age could not be determined robustly because of degeneracies in the CMD between age and metallicity. Armandroff \& Da Costa (1991) provided the crucial datum; they found that Pal 12, in spite of its large galactocentric distance, is quite metal-rich, obtaining $[\mathrm{Fe} / \mathrm{H}]=-0.6 \mathrm{dex}^{3}$ from low-resolution spectroscopy. More recently, Brown, Wallerstein, \& Zucker (1997) carried out a high-resolution spectroscopic study of two stars in Pal 12, which yielded $[\mathrm{Fe} / \mathrm{H}]=-1.0$ dex.

The combination of deep photometry and an estimate of $[\mathrm{Fe} / \mathrm{H}]$ led to the verification that $\mathrm{Pal} 12$ is indeed a young cluster. Rosenberg et al. (1998), as part of their recent study of the age dispersion within the Galactic GC system (Rosenberg et al. 1999), suggest an age for Pal 12 of roughly $70 \%$ that of the majority of the halo GCs. Assuming the latter group to be 12 Gyr old, they then infer an age of 8.4 Gyr for Pal 12, consistent with that of the earlier CMD studies of Gratton \& Ortolani (1988) and Stetson et al. (1989).

After the discovery of the Sagittarius dwarf spheroidal (dSph) galaxy by Ibata, Irwin, \& Gilmore (1994), several groups, including Layden \& Sarajedini (2000), noted that four Galactic GCs (M54, Arp 2, Terzan 7, and Terzan 8), based on

\footnotetext{
${ }^{1}$ Based in part on observations obtained at the W. M. Keck Observatory, which is operated jointly by the California Institute of Technology, the University of California, and the National Aeronautics and Space Administration

2 Palomar Observatory, MS 105-24, California Institute of Technology, Pasadena, CA 91125 jlc@astro.caltech.edu.

${ }^{3}$ The standard nomenclature is adopted; the abundance of element $\mathrm{X}$ is given by $\epsilon(\mathrm{X})=N(\mathrm{X}) / N(\mathrm{H})$ on a scale where $N(\mathrm{H})=10^{12} \mathrm{H}$ atoms. Then $[\mathrm{X} / \mathrm{H}]=\log [N(\mathrm{X}) / N(\mathrm{H})]-\log [N(\mathrm{X}) / N(\mathrm{H})]_{\odot}$ and similarly for $[\mathrm{X} / \mathrm{Fe}]$.
}

their positions on the sky, appear to form a stream extending from the Sgr dSph, and they suggested that these GCs had been tidally stripped away from the Sgr galaxy. The GC M54 was postulated to be the original nucleus of the Sgr galaxy.

Irwin (1999) was the first to suggest that the GC Pal 12 had also been tidally captured from the Sgr dSph galaxy by our Galaxy. Dinescu et al. (2000) measured the proper motion of this GC and calculated its orbit to find that Pal 12's tidal capture from the Sgr dSph took place about $1.7 \mathrm{Gyr}$ ago. Ibata et al. (2001) demonstrated the existence of an extended tidal stream of debris from the Sgr dSph galaxy in the Galactic halo, which has recently been detected in the Two Micron All Sky Survey (2MASS; Majewski et al. 2003) and in the Sloan Digital Sky Survey (Ivezić et al. 2003) databases. Deep optical imaging over wide fields around Pal 12 by Martinez-Delgado et al. (2002) and by Bellazzini et al. (2003) show that this GC is embedded in the extended debris stream of stars torn from the Sgr dSph.

The only existing high-dispersion spectroscopic study of Pal 12 (that of Brown et al. 1997) was hitting the limits of what was observationally possible with a $4 \mathrm{~m}$ telescope. They analyzed the spectra of only the two brightest probable members. Their primary result was that Pal 12 did not appear to show the enhancement of the $\alpha$-process elements seen in almost all GC stars. Although this is extremely interesting, the accuracy of their analysis was limited by the quality of their spectra and some key elements were not included. The purpose of the present paper is to provide a firmer foundation for their results, to extend them as possible, and to compare the properties of the Pal 12 stars with the recently published abundance distributions for stars in the Sgr dSph galaxy of Bonifacio et al. (2000), Smecker-Hane \& McWilliam (2003), McWilliam, Rich, \& Smecker-Hane (2003), and Bonifacio et al. (2003).

\section{STELLAR SAMPLE AND STELLAR PARAMETERS}

Since Pal 12 is potentially a young cluster in the outer halo, there have been, as noted above, several recent CMD studies 
of Pal 12. This is a rather sparse cluster, and there are only four probable members on the red giant branch (RGB) brighter than $V \sim 16 \mathrm{mag}$. These are the four stars we have observed; they are the same four stars that were observed at low resolution in the region of the IR Ca triplet by Armandroff \& Da Costa (1991). The stellar identifications we adopt are those of Harris \& Canterna (1980).

As in our earlier papers, we use the $V-J$ and $V-K$ colors to establish the $T_{\text {eff }}$ for these stars. We use the grid of predicted broadband colors and bolometric corrections of Houdashelt, Bell, \& Sweigart (2000) based on the MARCS stellar atmosphere code of Gustafsson et al. (1975). In Cohen, Behr, \& Briley (2001), we demonstrated that the Kurucz and MARCS predicted colors are essentially identical, at least for the specific colors used here. The optical photometry we adopt is from Stetson et al. (1989), and the infrared photometry is from 2MASS (Skrutskie et al. 1997). The reddening is low; we adopt $E(B-V)=0.02 \mathrm{mag}$ and a distance of $19.1 \mathrm{kpc}$ from the on-line database of Harris (1996); the all-sky maps of Schlegel, Finkbeiner, \& Davis (1998) yield a slightly larger $E(B-V)$ of $0.036 \mathrm{mag}$. It is important to note that the distance was obtained with a knowledge of the probable young age of this anomalous GC.

The surface gravities for the Pal 12 red giants are calculated from their observed $V$ magnitudes, their $T_{\text {eff }}$, the cluster distance and the reddening, as in our earlier papers. Here, however, based on the isochrones of Yi et al. (2001), we adopt a mass for the RGB stars of $1.0 M_{\odot}$ rather than the $0.8 M_{\odot}$ used in our earlier GC abundance analyses; the latter is appropriate for metal-poor clusters with an age of $12 \mathrm{Gyr}$, but not for Pal 12.

The most luminous Pal 12 giant, star S1, is somewhat cooler than the minimum $T_{\text {eff }}$ in the grid of Houdashelt et al. (2000), and extrapolation beyond the limit of this color grid was required. The nominal $T_{\text {eff }}$ of $3850 \mathrm{~K}$ so inferred from its observed colors gave poor ionization equilibrium. The photometric errors for our data produce a $\pm 50 \mathrm{~K}$ uncertainty in $T_{\text {eff }}$, hence we adopt a $T_{\text {eff }}$ for this star of $3900 \mathrm{~K}$. The resulting stellar parameters are listed in Table 1 . The $T_{\text {eff }}$ for the two stars analyzed by Brown et al. (1997; the cooler of the four stars analyzed here) are $\sim 60 \mathrm{~K}$ higher than those adopted here; they use $V-I$ photometry in one case and a $K$ magnitude for star S1 from Cohen, Frogel, \& Persson (1978); the older photometry they use surely has uncertainties at least as large as the photometry we use. Given that, the agreement in $T_{\text {eff }}$ for the two stars in common seems reasonable.

\section{OBSERVATIONS}

All spectra were obtained with HIRES (Vogt et al. 1994) at the Keck Observatory. The four Pal 12 stars are too far apart on the sky to fit two within the allowed HIRES slit length, and

TABLE 1

Stellar Parameters for the Palomar 12 Sample

\begin{tabular}{|c|c|c|c|}
\hline $\mathrm{ID}^{\mathrm{a}}$ & $\begin{array}{l}T_{\text {eff }} \\
(\mathrm{K})\end{array}$ & $\begin{array}{l}\log g \\
(\operatorname{dex})\end{array}$ & $\begin{array}{c}v_{t} \\
\left(\mathrm{~km} \mathrm{~s}^{-1}\right)\end{array}$ \\
\hline 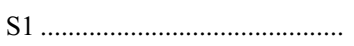 & 3900 & 0.63 & 1.8 \\
\hline 1118 & 4000 & 0.84 & 1.8 \\
\hline 1128 & 4260 & 1.30 & 1.7 \\
\hline 1305 & 4465 & 1.62 & 1.7 \\
\hline
\end{tabular}

${ }^{a}$ Identifications are from Harris \& Canterna 1980. hence each had to be observed individually. The HIRES configuration used a 1"1 wide slit (spectral resolution 34,000). Spectral coverage extended from 4650 to $7010 \AA$, with small gaps in coverage between the echelle orders because of the current undersized HIRES detector.

The spectra were exposed to a signal-to-noise ratio exceeding 100 per 4 pixel resolution element in the continuum at the center of order 64 (about $5670 \AA$ ). This was calculated assuming Poisson statistics and ignoring issues of cosmic-ray removal, flattening etc. These spectra were reduced using a combination of Figaro scripts (Shortridge 1993) and the software package MAKEE. ${ }^{4}$ Details of the exposures are given in Table 2. Heliocentric radial velocities were measured as described in Ramírez \& Cohen (2002); all the stars are confirmed as members of Pal 12. The four stars have a mean heliocentric $v_{r}$ of $+28.9 \mathrm{~km} \mathrm{~s}^{-1}$, with $\sigma=0.8 \mathrm{~km} \mathrm{~s}^{-1}$, consistent with the presumed low mass of this cluster. (The observational error has not been removed from this observed velocity dispersion.)

The search for absorption features present in our HIRES data and the measurement of their equivalent width $\left(W_{\lambda}\right)$ was done automatically with a FORTRAN code, EWDET, developed for our GC project. Details of this code and its features are described in Ramírez et al. (2001). Since we are observing only the most luminous (i.e., the coolest) stars in a highmetallicity cluster, considerable hand checking of the equivalent widths had to be done at various stages of the analysis.

A list of unblended atomic lines with atomic parameters was created by merging our existing GC list, developed from our earlier work on M71 and M5, adding in bluer lines in part from our work on very metal poor stars and in part as required to fill in the bluer orders covered here. We made extensive use of the NIST Atomic Spectra Database, Version 2.0 (NIST Standard Reference Database No. 78, see Wiese, Smith, \& Miles 1969; Martin, Fuhr, \& Wiese 1988; Fuhr, Martin, \& Wiese 1988; Wiese, Fuhr, \& Deters 1996). The online solar spectrum taken with the Fourier transform spectroscope at the National Solar Observatory of Wallace, Hinkle, \& Livingston $(1998)^{5}$ and the set of solar line identifications of Moore, Minnaert, \& Houtgast (1966) were also used. The list of lines identified and measured by EWDET is then correlated, taking the radial velocity into account, to the list of suitable unblended lines to specifically identify the various atomic lines.

All lines with $W_{\lambda}$ exceeding $200 \mathrm{~m} \AA$ were rejected, except for the 6141.7 and $6496.9 \AA$ lines of $\mathrm{Ba}$ II. The even stronger $4934 \AA$ line of $\mathrm{Ba}$ II was eliminated; it is blended and its $W_{\lambda}$ exceeded the cutoff in all the sample stars. The list of equivalent widths used in the this analysis for each of the four stars in Pal 12 is given in Table 3.

To the maximum extent possible, the atomic data and the analysis procedures used here are identical to those developed in our earlier papers on M71 and M5 (Cohen et al. 2001; Ramírez et al. 2001; Ramírez \& Cohen 2002; Ramírez \& Cohen 2003). For ions with hyperfine structure (HFS), we synthesize the spectrum for each line including the appropriate HFS and isotopic components. We use the HFS components from Prochaska et al. (2000) for the lines of Sc II, V I, Mn I, and Co I. For Ba II, we adopt the HFS from McWilliam (1998). We use the laboratory spectroscopy of Lawler, Bonvallet, \&

\footnotetext{
${ }^{4}$ MAKEE was developed by T. A. Barlow specifically for reduction of Keck HIRES data. It is freely available on the World Wide Web at the Keck Observatory home page (http://www2.keck.hawaii.edu:3636/).

${ }^{5}$ See ftp://ftp.noao.edu.fts/visatl/README.
} 
TABLE 2

Sample of Stars in Palomar 12

\begin{tabular}{|c|c|c|c|c|c|}
\hline $\mathrm{ID}^{\mathrm{a}}$ & $\begin{array}{c}V^{\mathrm{b}} \\
(\mathrm{mag})\end{array}$ & Date Obs. & $\begin{array}{l}\text { Exp. Time } \\
\text { (s) }\end{array}$ & $\mathrm{S} / \mathrm{N}^{\mathrm{c}}$ & $\begin{array}{c}v_{r} \\
\left(\mathrm{~km} \mathrm{~s}^{-1}\right)\end{array}$ \\
\hline . & 14.57 & 2003 Jun & 1500 & $>100$ & +28.8 \\
\hline 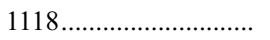 & 14.84 & 2003 Jun & 1800 & $>100$ & +30.0 \\
\hline 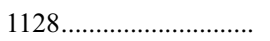 & 15.43 & 2003 Jun & 1500 & $>100$ & +28.1 \\
\hline 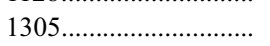 & 15.86 & 2003 Aug & 3600 & $>100$ & +28.8 \\
\hline
\end{tabular}

${ }^{a}$ Identifications are from Harris \& Canterna 1980.

${ }^{b} V$ photometry from Stetson et al. 1989.

${ }^{c}$ Signal-to-noise ratio in the continuum near $5865 \AA$ A per 4 pixel spectral resolution element.

Sneden (2001a) and Lawler et al. (2001b) to calculate the HFS patterns for $\mathrm{La}$ II and for $\mathrm{Eu}$ II. We have updated our $\mathrm{Nd}$ II $g f$ values to those of Den Hartog et al. (2003).

We use the solar abundances of Grevesse \& Sauval (1998), modified for the special cases of $\mathrm{La}$ II, Nd II, and Eu II with those found by the respective recent laboratory studies cited above. In particular, this means we have adopted $\epsilon(\mathrm{Fe})$ for the Sun of 7.52 dex, although in our earlier papers on M71 and M5 we used 7.44 dex for $\epsilon(\mathrm{Fe})(\mathrm{Sun})$.

The microturbulent velocity $\left(v_{t}\right)$ of a star can be determined spectroscopically by requiring the abundance to be independent of the strength of the lines. We apply this technique here to the large sample of detected $\mathrm{Fe}$ I lines in each star and use $v_{t}=1.7$ or $1.8 \mathrm{~km} \mathrm{~s}^{-1}$ for the four Pal 12 stars.

\section{ABUNDANCE RESULTS FOR Pal 12}

Given the derived stellar parameters from Table 1, we determined the abundances using the equivalent widths obtained as described above. The abundance analysis is carried out using a current version of the LTE spectral synthesis program MOOG (Sneden 1973). We employ the grid of stellar atmospheres from Kurucz (1993) with a metallicity of $[\mathrm{Fe} / \mathrm{H}]=$ $-1.0 \mathrm{dex}^{6}$ to compute the abundances of $\mathrm{O}, \mathrm{Na}, \mathrm{Mg}, \mathrm{Si}, \mathrm{Ca}$, $\mathrm{Sc}, \mathrm{Ti}, \mathrm{V}, \mathrm{Cr}, \mathrm{Mn}, \mathrm{Fe}, \mathrm{Co}, \mathrm{Ni}, \mathrm{Cu}, \mathrm{Zn}, \mathrm{Y}, \mathrm{Zr}, \mathrm{Ba}, \mathrm{La}, \mathrm{Nd}$, and $\mathrm{Eu}$ using the four stellar atmosphere models with the closest $T_{\text {eff }}$ and $\log g$ to each star's parameters. The abundances were interpolated using results from the closest stellar model atmospheres to the appropriate $T_{\text {eff }}$ and $\log g$ for each star given in Table 1. The results for the abundances of these species in the four stars in Pal 12 are given in Table 4.

Table 6 of Ramírez \& Cohen (2002) (which discusses M71, a GC of similar overall abundance) is a sensitivity table presenting the changes in deduced abundances of various species for small changes in $T_{\mathrm{eff}}, \log g$, and $v_{i}$; the entries in this table for the cooler stars in M71 ( $\left.T_{\text {eff }}=4250 \mathrm{~K}\right)$ can be used for the Pal 12 stars as well.

\footnotetext{
${ }^{6}$ We use the grid of models without convective overshoot.
}

The ionization equilibrium for both Fe I versus Fe II and for $\mathrm{Ti}$ I versus $\mathrm{Ti}$ II is satisfactory. The average difference for the four stars in $\mathrm{Pal} 12$ between $[\mathrm{Fe} / \mathrm{H}]$ as inferred from $\mathrm{Fe}$ II lines and from $\mathrm{Fe}$ I lines is $+0.11 \pm 0.07 \mathrm{dex}$ and $+0.16 \pm 0.07 \mathrm{dex}$ for $\mathrm{Ti}^{7}$ The $\mathrm{Fe}$ ionization equilibrium shifts by 0.2 dex for a $100 \mathrm{~K}$ change in $T_{\text {eff }}$ in this temperature regime, hence a systematic increase of the adopted $T_{\text {eff }}$ values by $50 \mathrm{~K}$ would eliminate these small discrepancies. This possible systematic offset, for which no correction has been made, is slightly smaller than the uncertainty in $T_{\text {eff. }}$. It might result from adopting a reddening for Pal 12 that is slightly too small.

Following upon our previous work, no non-LTE corrections have been applied for the specific ions studied in the Pal 12 stars. The detailed non-LTE calculations of Gratton et al. (1999) and of Takeda et al. (2003) for the two Na I doublets we use suggest that for this regime of $T_{\text {eff }}$ the non-LTE correction is about +0.15 dex. For Ba II, the non-LTE calculations of Mashonkina \& Gehren (2000) and of Mashonkina, Gehren, \& Bikmaev (1999) suggest that a non-LTE correction of -0.15 dex is appropriate for the metallicity of Pal 12 and the set of $\mathrm{Ba}$ II lines we used. In comparing with other abundance analyses, the issue of implementing non-LTE corrections and their adopted magnitudes must be considered.

\subsection{Comments on Individual Elements}

The oxygen abundance is derived from the forbidden lines at 6300 and $6363 \AA$. The subtraction of the night-sky emission lines at these wavelengths was reasonably straightforward. The stellar [ $\left.\mathrm{O}_{\mathrm{I}}\right]$ lines in the spectra of the Pal 12 stars are strong enough and the radial velocity of Pal 12 is sufficiently different from $0 \mathrm{~km} \mathrm{~s}^{-1}$ (and, for the faintest Pal 12 star observed, Pal 12 star 1305, the heliocentric correction provides additional help in separating the separating the stellar and atmospheric [O I] lines) that their $W_{\lambda}$ can be reliably measured. The $\mathrm{C} / \mathrm{O}$ ratio was assumed to be solar. The $\mathrm{O}$ abundance is given with respect to $[\mathrm{Fe} / \mathrm{H}]$ deduced from lines of $\mathrm{Fe} \mathrm{I}$; the

\footnotetext{
${ }^{7}$ Due to the possible presence of random errors in stellar parameters, all abundance ratios are assigned a minimum uncertainty of 0.05 dex.
}

TABLE 3

Equivalent Widths for the Palomar 12 Stars

\begin{tabular}{|c|c|c|c|c|c|c|c|}
\hline Ion & $\begin{array}{c}\lambda \\
(\AA)\end{array}$ & $\begin{array}{c}\chi \\
(\mathrm{eV})\end{array}$ & $\log g f$ & $\begin{array}{c}W_{\lambda}(\mathrm{S} 1) \\
(\mathrm{m} \AA)\end{array}$ & $\begin{array}{c}W_{\lambda}(1118) \\
(\mathrm{m} \AA)\end{array}$ & $\begin{array}{c}W_{\lambda}(1128) \\
(\mathrm{m} \AA)\end{array}$ & $\begin{array}{c}W_{\lambda}(1305) \\
(\mathrm{m} \AA)\end{array}$ \\
\hline \multirow[t]{2}{*}{$\mathrm{O}_{\text {I }} \ldots \ldots \ldots \ldots \ldots$} & 6300.30 & 0.00 & -9.78 & 68 & 79 & 39 & 39 \\
\hline & 6363.78 & 0.02 & -10.30 & 45 & 40 & 14 & 18 \\
\hline \multirow[t]{3}{*}{ 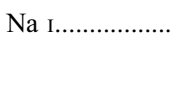 } & 5682.63 & 2.10 & -0.70 & 113 & 103 & 77 & 67 \\
\hline & 5688.19 & 2.10 & -0.42 & 128 & 108 & 100 & 94 \\
\hline & 6154.23 & 2.10 & -1.53 & 41 & 35 & 19 & 15 \\
\hline
\end{tabular}


TABLE 4

Derived Abundances for Palomar 12

\begin{tabular}{|c|c|c|c|c|c|c|c|c|c|c|c|c|}
\hline \multirow[b]{2}{*}{ Ion } & \multicolumn{3}{|c|}{$\mathrm{S} 1$} & \multicolumn{3}{|c|}{1118} & \multicolumn{3}{|c|}{1128} & \multicolumn{3}{|c|}{1305} \\
\hline & $\begin{array}{c}{[\mathrm{X} / \mathrm{Fe}]} \\
(\mathrm{dex})\end{array}$ & $\begin{array}{c}\sigma^{\mathrm{a}} \\
(\mathrm{dex})\end{array}$ & No. Lines & $\begin{array}{c}{[\mathrm{X} / \mathrm{Fe}]} \\
(\mathrm{dex})\end{array}$ & $\begin{array}{c}\sigma^{\mathrm{a}} \\
(\mathrm{dex})\end{array}$ & No. Lines & $\begin{array}{c}{[\mathrm{X} / \mathrm{Fe}]} \\
(\mathrm{dex})\end{array}$ & $\begin{array}{c}\sigma^{\mathrm{a}} \\
(\operatorname{dex})\end{array}$ & No. Lines & $\begin{array}{c}{[\mathrm{X} / \mathrm{Fe}]} \\
(\mathrm{dex})\end{array}$ & $\begin{array}{c}\sigma^{a} \\
(\operatorname{dex})\end{array}$ & No. Lines \\
\hline $\mathrm{O}_{\mathrm{I}} \ldots \ldots \ldots . .$. & 0.04 & 0.04 & 2 & 0.21 & 0.19 & 2 & -0.06 & 0.21 & 2 & 0.07 & 0.13 & 2 \\
\hline $\mathrm{Na}$ I.......... & -0.47 & 0.18 & 4 & -0.52 & 0.16 & 4 & -0.55 & 0.20 & 4 & -0.49 & 0.16 & 4 \\
\hline Mg I........ & -0.14 & $\ldots$ & 1 & 0.07 & 0.11 & 2 & 0.09 & 0.30 & 2 & 0.09 & 0.18 & 3 \\
\hline Si I .......... & 0.14 & 0.16 & 12 & 0.03 & 0.16 & 13 & 0.11 & 0.18 & 13 & 0.10 & 0.14 & 13 \\
\hline $\mathrm{Ca}$ I......... & -0.17 & 0.24 & 13 & -0.25 & 0.22 & 14 & -0.18 & 0.19 & 14 & -0.16 & 0.15 & 14 \\
\hline $\mathrm{Sc}$ II ........ & -0.16 & 0.17 & 6 & -0.12 & 0.16 & 6 & -0.08 & 0.16 & 7 & -0.07 & 0.17 & 7 \\
\hline Ti I.......... & -0.10 & 0.21 & 26 & -0.17 & 0.16 & 28 & -0.12 & 0.25 & 33 & -0.12 & 0.14 & 30 \\
\hline Ti II .......... & 0.02 & 0.25 & 4 & -0.03 & 0.13 & 5 & 0.08 & 0.18 & 6 & 0.08 & 0.10 & 5 \\
\hline $\mathrm{V}_{\mathrm{I}} \ldots \ldots \ldots$ & -0.31 & 0.19 & 8 & -0.41 & 0.20 & 9 & -0.35 & 0.15 & 10 & -0.38 & 0.14 & 10 \\
\hline $\mathrm{Cr}_{\mathrm{I}} \ldots \ldots \ldots$ & 0.14 & 0.21 & 6 & 0.02 & 0.23 & 6 & -0.03 & 0.20 & 10 & 0.07 & 0.21 & 10 \\
\hline Mn I........ & -0.25 & 0.13 & 2 & -0.28 & 0.09 & 2 & -0.32 & 0.11 & 4 & -0.28 & 0.20 & 5 \\
\hline $\mathrm{Fe}_{\mathrm{I}}^{\mathrm{b}} \ldots \ldots$ & 6.76 & 0.18 & 123 & 6.72 & 0.23 & 131 & 6.70 & 0.19 & 146 & 6.72 & 0.19 & 146 \\
\hline Fe II ........ & 0.10 & 0.23 & 9 & 0.09 & 0.19 & 9 & 0.12 & 0.23 & 11 & 0.12 & 0.20 & 9 \\
\hline Co I.......... & -0.35 & 0.21 & 4 & -0.32 & 0.28 & 4 & -0.29 & 0.21 & 4 & -0.25 & 0.19 & 4 \\
\hline Ni I ......... & -0.11 & 0.17 & 21 & -0.21 & 0.17 & 22 & -0.20 & 0.19 & 23 & -0.20 & 0.18 & 21 \\
\hline $\mathrm{Cu}$ I.......... & -0.55 & 0.18 & 2 & 0.14 & 0.16 & 2 & -1.01 & 0.27 & 2 & -0.69 & 0.07 & 2 \\
\hline $\mathrm{Zn}$ I......... & -0.45 & $\ldots$ & 1 & -0.64 & 0.16 & 2 & -0.51 & 0.18 & 2 & -0.38 & 0.06 & 2 \\
\hline Y II ......... & -0.16 & 0.93 & 2 & -0.60 & 0.52 & 2 & -0.49 & 0.36 & 2 & -0.35 & 0.17 & 3 \\
\hline Zr I........... & -0.16 & 0.07 & 3 & -0.28 & 0.06 & 3 & -0.22 & 0.05 & 3 & -0.16 & 0.06 & 3 \\
\hline Ba II ........ & 0.28 & 0.05 & 3 & 0.27 & 0.05 & 3 & 0.25 & 0.06 & 3 & 0.27 & 0.08 & 3 \\
\hline La II ........ & 0.25 & 0.06 & 2 & 0.22 & 0.01 & 2 & 0.31 & 0.08 & 2 & 0.09 & 0.02 & 2 \\
\hline Nd II ....... & 0.27 & $\ldots$ & 1 & 0.38 & 0.26 & 2 & 0.28 & 0.06 & 2 & 0.40 & 0.05 & 2 \\
\hline $\mathrm{Eu}$ II ......... & 0.62 & $\ldots$ & 1 & 0.58 & $\ldots$ & 1 & 0.55 & $\ldots$ & 1 & 0.69 & $\ldots$ & 1 \\
\hline
\end{tabular}

a This is the $1 \sigma \mathrm{rms}$ deviation of the set of abundances derived from each of the observed absorption lines about the mean abundance.

b For Fe I only, we give $[\mathrm{Fe} / \mathrm{H}]$.

mean $[\mathrm{O} / \mathrm{Fe}]$ becomes 0.09 dex smaller if expressed using the Fe II lines instead. The IR triplet at $7770 \AA$ is beyond the wavelength range of these spectra. No corrections were made for the Ni I blend in the $6300 \AA$ discussed by Allende Prieto, Lambert, \& Asplund (2001); when this and the small difference in adopted $g f$ value are taken into account, their value for the solar $\mathrm{O}$ abundance agrees with that adopted here to within 0.05 dex.

No lines of $\mathrm{Al}$ can be reached with this HIRES configuration; the $6697 \AA$ double unfortunately falls in an interorder gap.

There are two detected lines of $\mathrm{Cu}$, both of which have very large HFS corrections, ranging between -0.5 and -1.0 dex. The abundance of copper in Pal 12 is therefore quite uncertain.

Three lines of $Y_{\text {II }}$ are used here. They are all crowded, and the line at $5205.4 \AA$ is too blended to use in all but the hottest star. The $g f$ values are from Hannaford et al. (1982) and give good results for the Sun. In the hottest star, the three lines gives reasonably consistent results. However, for star S1, the coolest star, the $4883.7 \AA$ line gives an abundance more than a factor of 10 higher than the $5087.4 \AA$ line. We are not using any HFS corrections for Y. Hannaford et al. (1982) suggest they are small, but perhaps the $\mathrm{Y}$ lines are so strong in this star that use of HFS is required. In computing the mean $\mathrm{Y}$ abundance for Pal 12, we ignore star S1.

\subsection{Abundance Spreads}

We calculate the mean abundance for each atomic species (X) with observed absorption lines for the four stars in Pal 12, as well as the $1 \sigma$ rms value about that mean. These values are given in the first three columns of Table 5. This is compared with the observational uncertainty $[\sigma(\mathrm{obs})]$, given in the fourth column of the table. The variable $\sigma(\mathrm{obs})$ is taken as the uncertainty of the mean abundance for a single star, i.e., the $1 \sigma$ rms value about the mean abundance of species $\mathrm{X}$ in a star divided by $\sqrt{N}$, where $N$ is the number of observed lines of species $\mathrm{X}$. Values of $N$ can be found in Table 4. This definition of the observational uncertainty presumes that errors in the $W_{\lambda}$ and the atomic data dominate; random errors in $T_{\text {eff }}$ or $\log g$ are not included. Some species, an example being $\mathrm{Fe}$ I with its very large value of $N$, have very small values of $\sigma(\mathrm{obs})$; we adopt a minimum of $0.05 \mathrm{dex}$ for this parameter.

The ratio of these two different $\sigma$ values is an indication of whether there is any intrinsic star-to-star variation in $[\mathrm{X} / \mathrm{Fe}]$. A high value of this "spread ratio" (SR), tabulated in the next to last column of this table, suggests a high probability of intrinsic scatter for the abundance of the species X.

The spectrum of the coolest Pal 12 star (S1) is the most crowded and blended and also has many lines whose $W_{\lambda}$ exceeds the cutoff value of $200 \mathrm{~m} \AA$. For three species, this reduces the number of detected lines to only a single line in this star, while the other three Pal 12 stars in our sample have two or more detected lines. Thus, in the case of $\mathrm{Mg}_{\mathrm{I}}, \mathrm{Zn} \mathrm{I}$, and $\mathrm{Nd}$ II (as well as Y II, see above), the averages and other statistics given in Table 5 are calculated using only three stars, ignoring the coolest one.

Inspection of Table 5 shows that for most species $0.6<\mathrm{SR} \leq 1.0$, indicating no sign of an intrinsic star-to-star range in abundance. Only for $\mathrm{O}, \mathrm{Cu}, \mathrm{Zn}, \mathrm{Zr}, \mathrm{La}$, and $\mathrm{Nd}$ does SR exceed 1.0. Copper has by far the largest calculated SR value; we believe that uncertainties associated with the large HFS corrections are responsible for the large spread in the derived $\mathrm{Cu}$ abundance among the four Pal 12 stars. Ignoring 
TABLE 5

Mean Abundances and Abundance Spreads for Four Stars in Palomar 12

\begin{tabular}{|c|c|c|c|c|c|}
\hline Species & $\begin{array}{c}\text { Mean Abund. } \\
{[\mathrm{X} / \mathrm{Fe}]} \\
(\operatorname{dex})\end{array}$ & $\begin{array}{c}\sigma \text { Around } \\
\text { Mean } \\
(\text { dex })\end{array}$ & $\begin{array}{c}\sigma(\mathrm{obs}) \\
(\mathrm{dex})\end{array}$ & $\begin{array}{c}\text { Spread } \\
\text { Ratio }^{\text {a }} \\
(\text { dex })\end{array}$ & $\begin{array}{l}\text { No. of } \\
\text { Stars }^{\text {b }}\end{array}$ \\
\hline 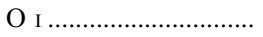 & 0.07 & 0.11 & 0.10 & 1.10 & 4 \\
\hline 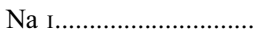 & -0.51 & 0.04 & 0.09 & 0.42 & 4 \\
\hline 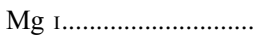 & 0.08 & 0.01 & 0.10 & 0.11 & 3 \\
\hline Si I & 0.10 & 0.05 & $0.05^{\mathrm{c}}$ & 0.90 & 4 \\
\hline 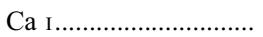 & -0.19 & 0.04 & 0.05 & 0.76 & 4 \\
\hline 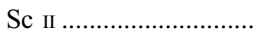 & -0.11 & 0.04 & 0.07 & 0.62 & 4 \\
\hline 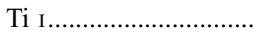 & -0.13 & 0.03 & $0.05^{\mathrm{c}}$ & 0.58 & 4 \\
\hline 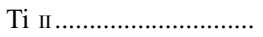 & 0.04 & 0.05 & 0.07 & 0.72 & 4 \\
\hline 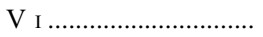 & -0.36 & 0.04 & 0.06 & 0.78 & 4 \\
\hline 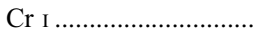 & 0.05 & 0.07 & 0.09 & 0.83 & 4 \\
\hline 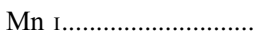 & -0.28 & 0.03 & 0.09 & 0.30 & 4 \\
\hline 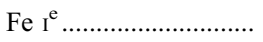 & 6.72 & 0.02 & $0.05^{\mathrm{c}}$ & 0.44 & 4 \\
\hline 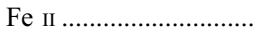 & 0.11 & 0.02 & 0.07 & 0.23 & 4 \\
\hline 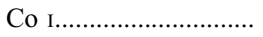 & -0.30 & 0.04 & 0.11 & 0.39 & 4 \\
\hline 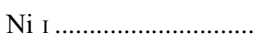 & -0.18 & 0.04 & $0.05^{\mathrm{c}}$ & 0.90 & 4 \\
\hline 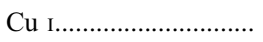 & -0.52 & 0.49 & 0.12 & $4.05^{\mathrm{d}}$ & 4 \\
\hline 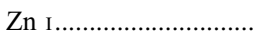 & -0.51 & 0.13 & 0.07 & 1.86 & 3 \\
\hline 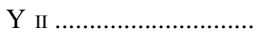 & $-0.48^{\mathrm{f}}$ & 0.12 & 0.19 & 0.67 & 3 \\
\hline 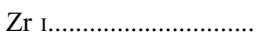 & -0.20 & 0.06 & $0.05^{\mathrm{c}}$ & 1.12 & 4 \\
\hline 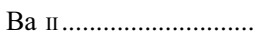 & 0.27 & 0.01 & $0.05^{\mathrm{c}}$ & 0.24 & 4 \\
\hline 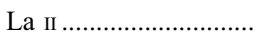 & 0.22 & 0.09 & $0.05^{\mathrm{c}}$ & 1.80 & 4 \\
\hline 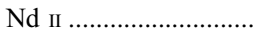 & 0.35 & 0.07 & 0.07 & 1.06 & 3 \\
\hline 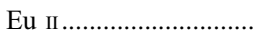 & 0.61 & 0.06 & $\ldots$ & $\ldots$ & 4 \\
\hline
\end{tabular}

a This is the ratio of $\sigma$ about the mean abundance for Pal 12 of the sample of four stars to $\sigma(\mathrm{obs})$.

b For some species, star S1, the coolest star in our Pal 12 sample, had significantly fewer usable lines, as the spectrum was more crowded and the lines became too strong to use.

c $\sigma$ (obs) is very low, in most cases due to the large number of lines used. It has been increased to 0.05 dex.

${ }^{\mathrm{d}} \mathrm{Cu}$ I has very large HFS corrections, between 0.5 and $1.0 \mathrm{dex}$.

e For Fe I only, we give $[\mathrm{Fe} / \mathrm{H}]$.

f The coolest star is excluded; see $\S 4.1$.

$\mathrm{Cu}$, all the remaining species listed above have only two detected lines at best (except for Zr I, which has three), many of the detected rare earth lines are weak, and some are crowded. We feel that when $N$ is very small $(N<4)$, our estimate of $\sigma(\mathrm{obs})$ is biased low, and hence the SR is biased high. Thus, there is no credible evidence from our data for an abundance spread for any species included in our analysis. In particular, the total range of $[\mathrm{Mg} / \mathrm{Fe}]$ is only 0.02 dex (excluding star $\mathrm{S} 1$, where only one $\mathrm{Mg}$ I line could be used), the total range of [Si/Fe] for all four Pal 12 stars is only 0.11 dex, etc.

Our derived $[\mathrm{Fe} / \mathrm{H}]$ for Pal 12 is 0.2 dex higher than that of Brown et al. (1997), who found $[\mathrm{Fe} / \mathrm{H}]=-1.0 \pm 0.1 \mathrm{dex}$. Only a small part $(\sim 0.05 \mathrm{dex})$ of that difference can be attributed to the cooler values of $T_{\text {eff }}$ adopted here. We add detections for the species $\mathrm{O}_{\mathrm{I}}, \mathrm{VI}$, and $\mathrm{Nd}$ II, not included in the previous work on this GC, and our abundances are much more precise than those of the previous study. We have a sample large enough to assess the possible presence of abundance spreads. To within their rather large errors, the Brown et al. (1997) abundance ratios agree with those we have derived. They correctly discerned the general nature of Pal 12's peculiarities.

\section{COMPARISON OF THE ABUNDANCES WITH THOSE OF OTHER STELLAR SYSTEMS}

In this section, we compare the abundance distribution of Pal 12 with those of various other galactic populations. These patterns reflect the history of star formation, the form of the initial mass function, and the gas flows within the Galaxy.
They provide signposts as to the nucleosynthesis in the early universe and the nature of the formation and collapse of the Galaxy. Since the abundance distribution of the stars in Pal 12 is so anomalous, we seek to find analogs of it within other stellar components of the Galaxy.

\subsection{Comparison with Galactic GCs}

We compare the abundance ratios we have derived for Pal 12 with those of typical Galactic GCs. We adopt M71 and M5 as representative Galactic GCs of suitable metallicity; Pal 12 has $[\mathrm{Fe} / \mathrm{H}]$ close to that of M71. We use the data from Ramírez \& Cohen (2002) for M71 and from Ramírez \& Cohen (2002) for M5; these previous analyses from our group are almost directly comparable with the present one for Pal 12 with no adjustments necessary. Figure 1 presents the abundance ratios as a function of atomic number for species between $\mathrm{O}$ and $\mathrm{Zr}$. Those of M71 and of M5 are shown in the bottom panel.

There are some features in common between the M71, M5, and Pal 12 stars, particularly the large odd-even effect, which refers to the unusually large deficiency of the odd atomic number species (including $\mathrm{Na}, \mathrm{Al}, \mathrm{Sc}, \mathrm{V}, \mathrm{Mn}$, and $\mathrm{Co}$ ) compared with their even atomic number neighbors in the regime from $\mathrm{Na}$ to $\mathrm{Ni}$ in the periodic table. ${ }^{8}$ However, there are also real and important differences. In particular, the $\alpha$ elements $\mathrm{Si}$,

\footnotetext{
${ }^{8}$ The solar abundances themselves with respect to $\mathrm{H},[\mathrm{X} / \mathrm{H}]$, show a strong odd-even effect, but what we are referring to here is an enhancement of this beyond what is characteristic of the solar composition.
} 


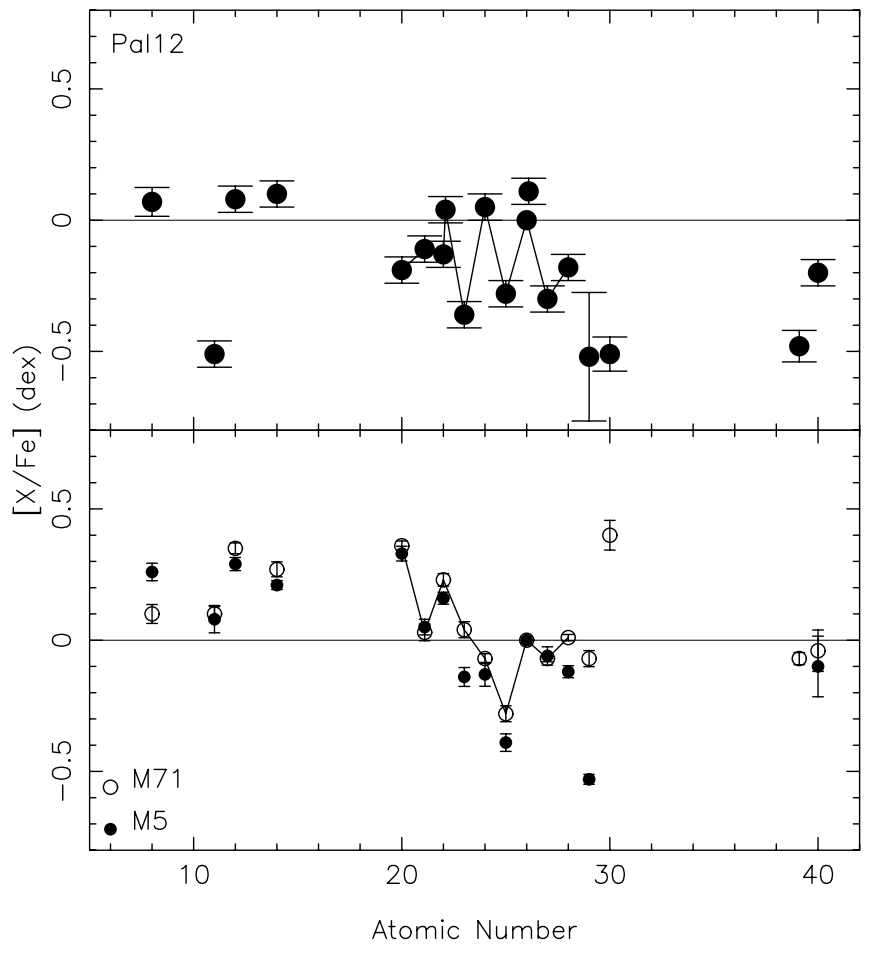

FIG. 1.-Top: Mean abundances of the four stars in Pal 12 as a function of atomic number from $\mathrm{O}$ through $\mathrm{Zr}$. The same information from large samples of stars in the GCs M71 and in M5 is shown using data from Ramírez \& Cohen (2002) and from Ramírez \& Cohen (2003). Those points representing the abundances of species of consecutive atomic number from 20 to 28 for Pal 12 and for M71 are connected by line segments.

$\mathrm{Ca}$, and $\mathrm{Ti}$, as well as $\mathrm{O}$, are substantially enhanced in M5 and in M71, as well as in essentially all GC stars, with $[\alpha / \mathrm{Fe}] \sim$ +0.3 dex. Pal 12, on the other hand, shows no enhancement of these elements, with the average of the mean values of $[\mathrm{Si} / \mathrm{Fe}]$, $[\mathrm{Ca} / \mathrm{Fe}]$, and $[\mathrm{Ti} / \mathrm{Fe}]$ being $-0.07 \pm 0.05 \mathrm{dex} ;[\mathrm{O} / \mathrm{Fe}]$ is also almost the solar value in Pal 12 (see Table 5). The deficiency of $\mathrm{Na}$ is very large in Pal 12 (the mean for the four Pal 12 stars of $[\mathrm{Na} / \mathrm{Fe}]$ is $-0.51 \pm 0.04 \mathrm{dex})$, much larger than is seen in Galactic GCs.

Figure 2 shows the heavy elements from $\mathrm{Y}$ to $\mathrm{Eu}$. The presence of enhanced $\mathrm{Eu}$ in $\mathrm{Pal} 12$ and in the comparison GCs M71 and M5 suggests a substantial contribution from the $r$-process. The $[\mathrm{Eu} / \mathrm{Nd}]$ and $[\mathrm{Eu} / \mathrm{La}]$ ratios in $\mathrm{Pal} 12$ are each within 0.2 dex of that of the solar $r$-process ratio determined by Burris et al. (2000) from the isotopic breakdowns of Käppeler, Beer, \& Wisshak (1989). However, Y and Zr show substantially larger deficiencies than are seen in Galactic GCs.

\subsection{Comparison with Galactic Field Stars in the Halo and in the Disk}

We compare the properties of Pal 12 with those of Galactic disk and halo stars. Reddy et al. (2003) have completed an extremely accurate and internally consistent analysis of $181 \mathrm{~F}$ and $\mathrm{G}$ dwarfs the vast majority of which belong to the Galactic thin disk. They cover the metallicity range $-0.7 \leq[\mathrm{Fe} / \mathrm{H}] \leq$ +0.2 . Their results are not consistent with the behavior of Pal 12, which is only slightly more metal-poor than the metalpoor end of their sample. While the behavior of the $\alpha$-elements $\mathrm{Si}, \mathrm{Ca}$, and Ti agree between the disk star sample and $\mathrm{Pal}$ 12, the behavior of $\mathrm{Na}$ is highly discrepant. In the disk stars, $[\mathrm{Na} / \mathrm{Fe}]$ rises from solar to about +0.15 dex at the metal-poor end of their sample, while that for Pal 12 is
$-0.51 \pm 0.04$ dex. The behavior of $[\mathrm{Mn} / \mathrm{Fe}]$ is consistent between the disk stars and Pal 12, but heavier than Fe, the agreement deteriorates again. The disk stars show $[\mathrm{Zn} / \mathrm{Fe}]$ rising to reach about +0.1 dex over this same range, while for Pal $12,[\mathrm{Zn} / \mathrm{Fe}]=-0.51 \pm 0.13 \mathrm{dex}$. $\mathrm{Y} / \mathrm{Fe}$ and $\mathrm{Eu} / \mathrm{Fe}$ retain their solar ratios throughout the metallicity range covered by Reddy et al. (2003), while those of Pal 12 are $-0.48 \pm$ 0.12 and $+0.61 \pm 0.06$, respectively.

A detailed study of a sample of stars from the thick disk has been carried out by Prochaska et al. (2000). These stars, which reach to lower metallicities than do the thin-disk stars, also show large enhancements of the $\alpha$ elements, with $\mathrm{Na}$ and $\mathrm{Zn}$ also enhanced. Thus, their abundance distribution also fails to match that of Pal 12.

Fulbright (2002) and Stephens (1999) have analyzed large samples of Galactic halo field stars. Fulbright (2002) attempted to correlate their kinematics with their abundance distributions. He uses the Galactic rest-frame velocities $\left(V_{\mathrm{RF}}\right)$, calculated from the $U V W$ velocities, removing the rotational velocity of the local standard of rest, to characterize the kinematic properties of the stars in his sample. Dinescu et al. (2000) have measured the proper motion of Pal 12 and suggest that the apogalactic radius for its orbit is $29.4 \pm 6.0 \mathrm{kpc}$. From their data, we calculate $V_{\mathrm{RF}}(\mathrm{Pal} 12)$ to be $251 \pm 35 \mathrm{~km} \mathrm{~s}^{-1}$.

Fulbright (2002) finds that the highest velocity stars in his sample have slightly lower $\alpha$-enhancements than are typical of most halo stars. A similar result was obtained by Stephens \& Boesgaard (2002), who concentrated on a sample of kinematically peculiar stars in the outer halo. These low $\alpha$-enhancements seen in a few stars that are probably in the outer Galactic halo are not as low as those we observe in Pal 12.

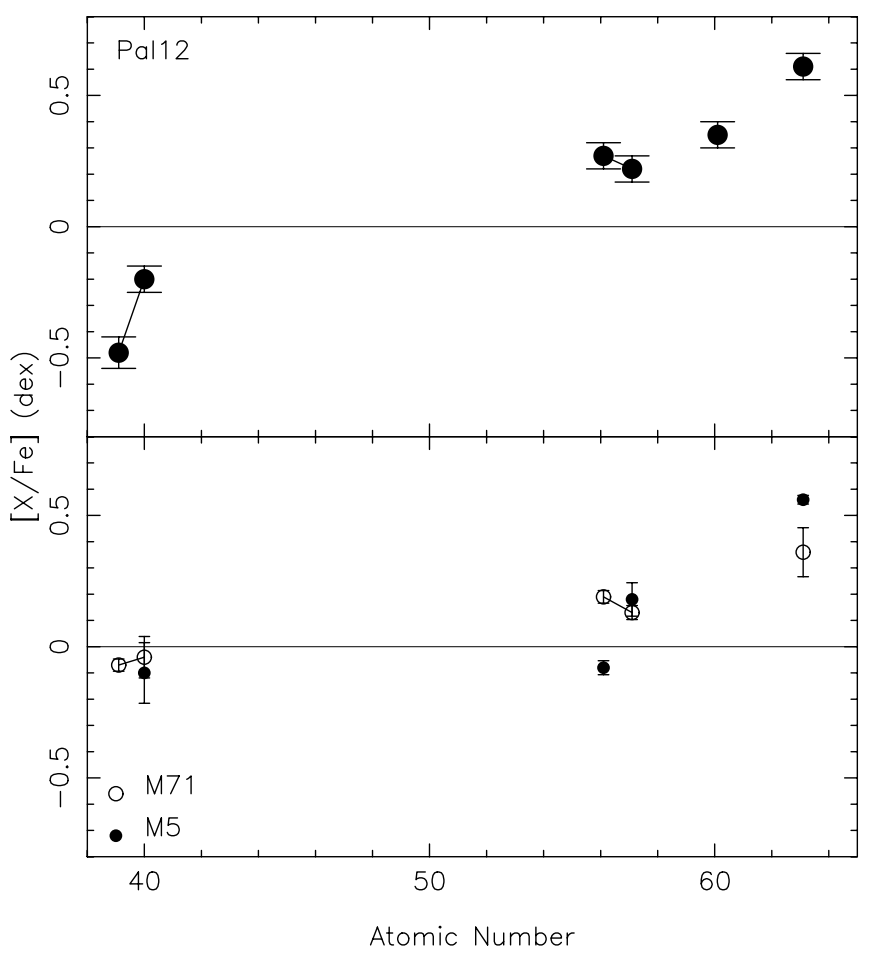

Fig. 2.-Top: Mean abundances of the four stars in Pal 12 as a function of atomic number from $\mathrm{Y}$ through $\mathrm{Eu}$. The same information from large samples of stars in the GCs M71 and in M5 is shown using data from Ramírez \& Cohen (2002) and from Ramírez \& Cohen (2003). Those points representing the abundances of species of consecutive atomic number for Pal 12 and for M71 are connected by line segments. 
The shift from the lighter to the heavier $s$-process elements between the first $s$-process (the $\mathrm{Zr}$ peak) peak toward the second peak at Ba seen in Pal 12 is also not matched by the halo stars. The trends for most element ratios with increasing $\left(V_{\mathrm{RF}}\right)$ found among halo stars by Fulbright (2002) in general have the right sign to reproduce the behavior of Pal 12 eventually, but fail to do so by significant amounts even for his highest velocity bin $\left(V_{\mathrm{RF}}>300 \mathrm{~km} \mathrm{~s}^{-1}\right)$.

There are a very small number of metal-poor Galactic halo stars known to have unusually low abundances of $\alpha$-elements, among the most extreme of which is $\mathrm{BD}+80^{\circ} 245$, which shows near-solar $\alpha / \mathrm{Fe}$ with $[\mathrm{Fe} / \mathrm{H}] \sim-2$ dex (Carney et al. 1997). However, even these stars, a small heterogeneous group of which were studied by Ivans et al. (2003), fail to match the abundance ratios seen among the Pal 12 stars.

\subsection{Comparison with the Sgr dSph Galaxy}

As shown above, the abundance distribution within Pal 12 fails to match that of typical Galactic GCs, most Galactic halo stars, and Galactic disk stars. We next see how well it matches that of stars within the Sgr dSph galaxy. We use the results of Bonifacio et al. (2000), who analyzed two stars, and of Smecker-Hane \& McWilliam (2003), who analyzed 14 Sgr stars. Results for $[\mathrm{Mn} / \mathrm{Fe}]$ from the latter sample are reported by McWilliam et al. (2003). These analyses of the Sgr stars are similar to ours in that they are classical LTE analyses and no corrections for non-LTE effects were made. Bonifacio et al. (2000) characterizes their results for two stars with $[\mathrm{Fe} / \mathrm{H}] \sim$ 0.2 dex by stating "the abundance ratios found are essentially solar with a few exceptions: Na shows a strong overdeficiency, the heavy elements $\mathrm{Ba}$ to $\mathrm{Eu}$ are overabundant, while $\mathrm{Y}$ is underabundant." This is a concise approximate description of the abundance ratios in Pal 12 .

We compare the mean $\alpha$ element ratio, $[\alpha / \mathrm{Fe}]$, defined as the average of $[\mathrm{Si} / \mathrm{Fe}],[\mathrm{Ca} / \mathrm{Fe}]$, and $[\mathrm{Ti} / \mathrm{Fe}]$, for stars in the $\mathrm{Sgr}$ $\mathrm{dSph}$ galaxy and for Pal 12. This is shown in the bottom panel of Figure 3 as a function of metallicity. The Sgr system at low metallicity behaves like typical Galactic GCs would, while at high metallicity, the $\alpha$-enhancement drops to zero. Bonifacio et al. (2003) analyze $\mathrm{O}, \mathrm{Mg}, \mathrm{Si}, \mathrm{Ca}$, and $\mathrm{Fe}$, but not $\mathrm{Ti}$, in 10 additional Sgr stars covering the range $-0.85<[\mathrm{Fe} / \mathrm{H}]<$ $+0.1 \mathrm{dex}$, and their results are similar. Pal 12 fits right on the trend defined by the Sgr stars when the comparison is made at the metallicity of Pal 12. The top panel shows the same for $[\mathrm{Na} / \mathrm{Fe}]$, where Pal 12 shares the tendency shown by the more metal-rich Sgr stars to show very large depletions of $\mathrm{Na}$. Also shown on this figure are the mean abundance ratios for a sample of five RGB stars in M54, a Galactic GC long believed to have been tidally stripped from the Sgr dSph galaxy, with data from Brown, Wallerstein, \& Gonzalez (1999).

The unusually low $\alpha$-ratios seen among the more metal-rich of the Sgr dSph stars are also seen in other dSph galaxies. Initial spectroscopic analyses from Keck/HIRES spectra for abundances in the Draco and the U Minor dSph galaxies have been carried out by Shetrone, Bolte, \& Stetson (1998) and by Shetrone, Côté, \& Sargent (2001), with a total sample of six stars in each of these two galaxies. In both of these galaxies, the $\alpha$-elements ( $\mathrm{Si}, \mathrm{Ca}, \mathrm{Ti}$ ) appear to be less enhanced relative to Fe than they are in the Galactic halo field. The four southern dSph galaxies with small samples of stars studied with UV-Visual Echelle Spectrograph (Sculptor, Fornax, Carina, and Leo I; Shetrone et al. 2003; Tolstoy et al. 2003) also show this pattern. Thus, this is now a well-established result for the metal-rich component of the dSph satellites of the Galaxy.

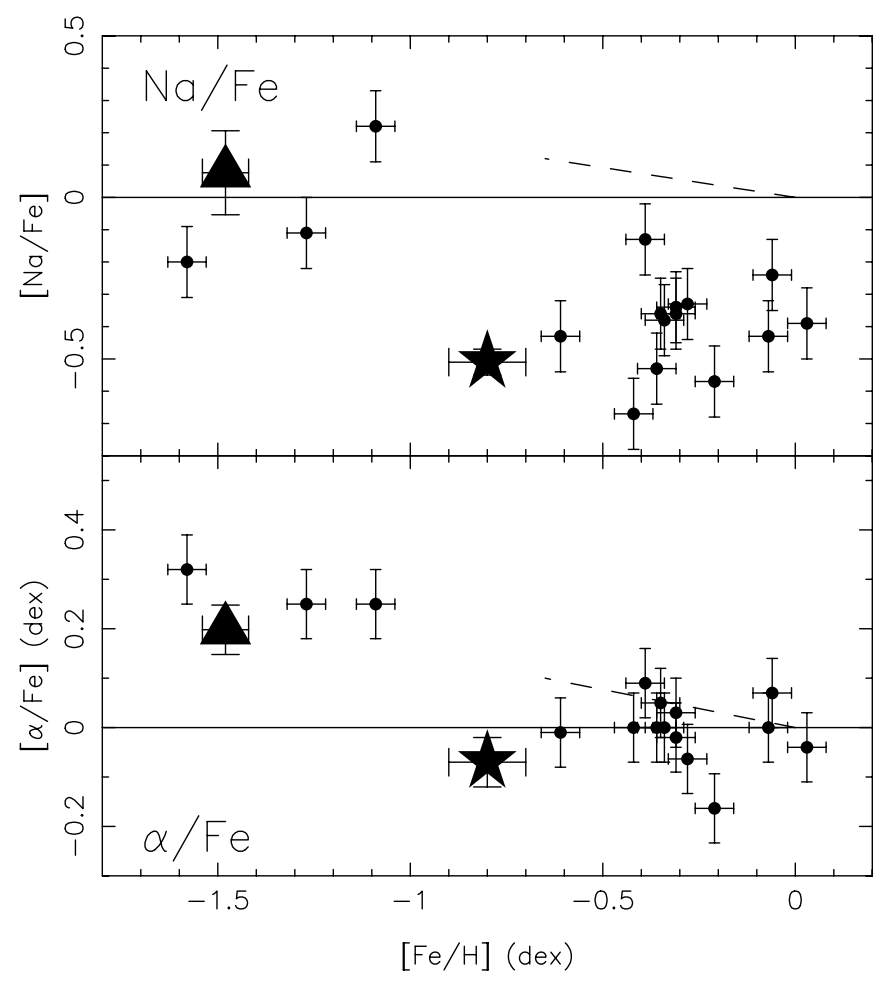

FIG. 3.-Mean abundance of the $\alpha$-elements $\mathrm{Si}, \mathrm{Ca}$, and $\mathrm{Ti}$ with respect to $\mathrm{Fe}$ as a function of $[\mathrm{Fe} / \mathrm{H}]$ for a sample of stars in the Sgr dSph galaxy with data from Bonifacio et al. (2000) and from Smecker-Hane \& McWilliam (2003). Our result for Pal 12 is indicated by the large star; that of the GC M54, indicated by the triangle, is taken from Brown et al. (1999). The dashed line represents the behavior of the thin disk stars from Reddy et al. (2003). Top: The same for $[\mathrm{Na} / \mathrm{Fe}]$.

Nissen \& Schuster (1997) suggested that the large depletion of $\mathrm{Na}$ seen in some $\alpha$-poor halo stars is accompanied by a smaller depletion of Ni. Nissen (2004) notes that this correlation extends to include stars in the Sgr and other local dSph galaxies, and again Pal 12 fits right on the trend at its metallicity. The possible origin of this odd coupling of a presumed $\alpha$-element with a presumed Fe-peak element is discussed in their papers.

Figure 4 shows the ratios $[\mathrm{La} / \mathrm{Fe}],[\mathrm{La} / \mathrm{Eu}]$, and $[\mathrm{La} / \mathrm{Y}]$ as a function of $[\mathrm{Fe} / \mathrm{H}]$ in a manner similar to that of Figure 3. Again, the Sgr stars show a systematic trend of each ratio with metallicity. In each panel of this figure, both the Pal 12 point and the M54 point lie on the trend at the metallicity appropriate for the GC. The shift of the $\mathrm{La} / \mathrm{Eu}$ ratio between lowand high-metallicity Sgr stars (with Pal 12 behaving like a low-metallicity Sgr star) is equivalent to the shift between $r$ - and $s$-process dominance among Galactic halo stars (see, e.g., Burris et al. 2000).

Among the set of four GCs long suspected to have been stripped from the Sgr dSph galaxy, Arp 2, like M54, is metalpoor. At low metallicity, both the Sgr stars and M54 show abundance ratios similar to typical Galactic GCs. It is only among the more metal-rich Sgr stars that differences emerge, which are shared by Pal 12. Terzan 7 is also suspected to be metal rich, but a preliminary study of three luminous RGB stars in this cluster by Wallerstein, Gonzalez, \& Geisler (2002) claimed to see the $\alpha$-enhancements typical of GCs. However, reanalysis of this material by Sbordone et al. (2003) and Tautvaisiene et al. (2004) gives $[\mathrm{Fe} / \mathrm{H}]=-0.6$ dex, with solar $\alpha / \mathrm{Fe}$, consistent with our Pal 12 result and with the run of the Sgr dSph stars. 


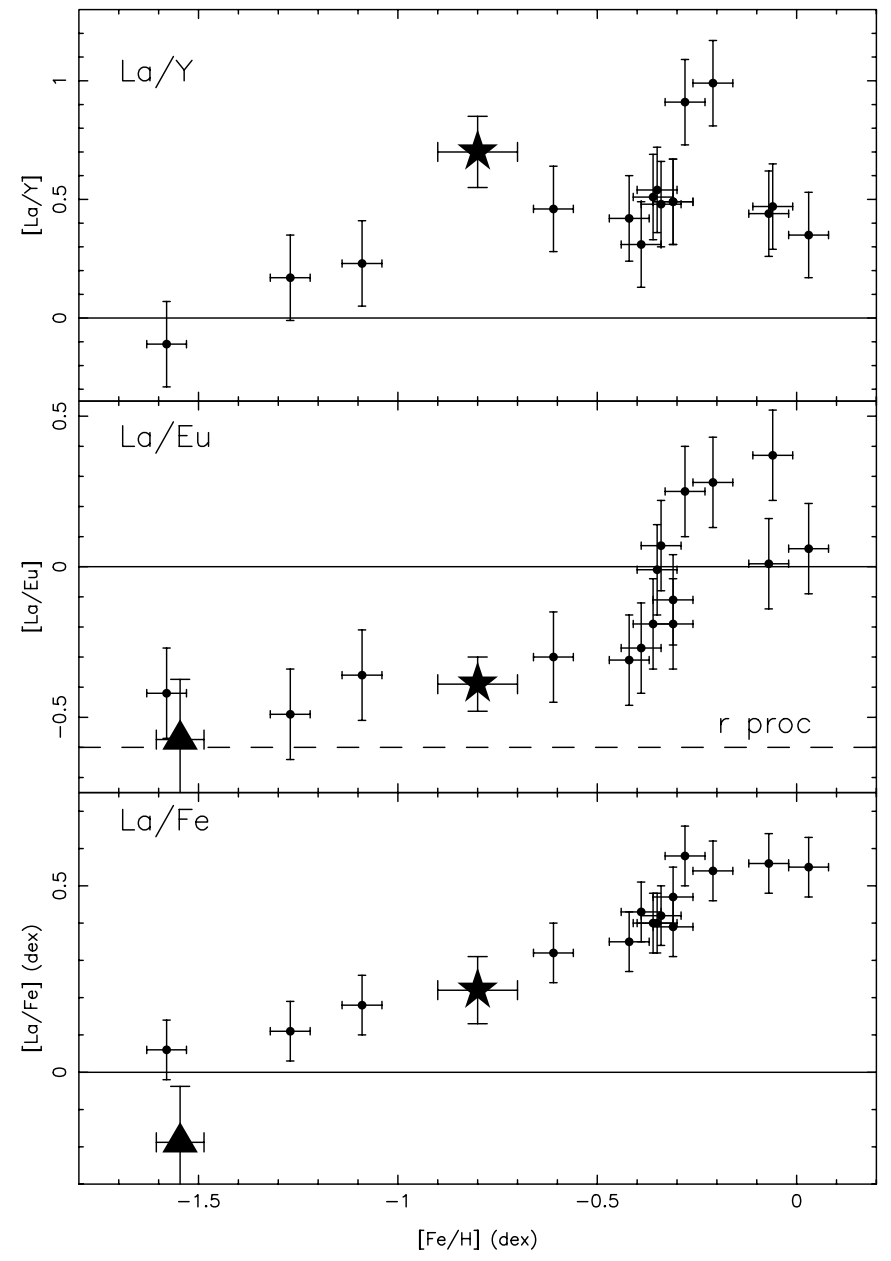

FIG. 4.-Bottom: Abundance ratio $[\mathrm{La} / \mathrm{Fe}]$ as a function of $[\mathrm{Fe} / \mathrm{H}]$ for a sample of stars in the Sgr dSph galaxy with data from Bonifacio et al. (2000) and from Smecker-Hane \& McWilliam (2003). Our result for Pal 12 is indicated by the large star; that of the GC M54, indicated by the triangle, is taken from Brown et al. (1999). Middle, the same for [La/Eu]; top, the same for $[\mathrm{La} / \mathrm{Y}]$. The dashed line in the middle panel indicates the $[\mathrm{La} / \mathrm{Eu}]$ ratio from the solar $r$-process.

\subsection{The Age-Metallicity Relation for Sgr}

Here we review the age-metallicity relation for the Sgr dSph galaxy and demonstrate that the measured metallicity and inferred age for Pal 12 are consistent with that to within the large uncertainties. Ages for GCs can be determined through isochrone fitting or through differences between the horizontal branch and the main-sequence turnoff from suitable multicolor photometry. The age of Pal 12 has been determined by Gratton \& Ortolani (1988), Stetson et al. (1989), and most recently by Rosenberg et al. (1998). Ages for the four GCs that have long been believed to be associated with the Sgr galaxy (M54, Arp 2, Terzan 7, and Terzan 8) are given by Layden \& Sarajedini (2000), as are the most recent abundance determinations for these objects. Smecker-Hane \& McWilliam (2003) have estimated ages for the 14 stars in their Sgr sample; these determinations for individual stars have much larger uncertainties than those for the GCs and are further compromised by the possible depth of the galaxy along the line of sight.

Figure 5 illustrates the age-metallicity (in the form of $[\alpha / \mathrm{H}])$ relation for the Sgr dSph stars with abundance analyses by Smecker-Hane \& McWilliam (2003) and for these four Galactic GCs, as well as for Pal 12. While the ages for the
Sgr stars are very uncertain, the location of Pal 12 in this figure appears consistent with the age-metallicity relationship displayed by the stars in the Sgr dSph galaxy. An analysis of more Sgr stars of varying ages and metallicities is needed to better define the age-metallicity relationship of the Sgr galaxy to refine this comparison.

\subsection{Comments on Nucleosynthesis}

The general principles of nucleosynthesis of the elements in stars are reviewed by Wheeler, Sneden, \& Truran (1989) and by McWilliam (1997). Some of the trends described above are fairly easy to explain in this context. The high $\alpha$-element ratios seen in Galactic GCs and halo stars are ascribed to a very old population for which there was insufficient time for Type I SN to have evolved and detonated and hence contributed their nuclear processed material (consisting mainly of Fe-peak elements) to the interstellar medium. The relevant minimum timescale for this is difficult to estimate since the evolution of close binary systems containing a white dwarf, believed to be the progenitors of Type Ia SN, must be followed in detail including mass accretion; a recent attempt to carry out this calculation by Han \& Podsiadlowski (2003) gives a minimum timescale of $\sim 3 \times 10^{8}$ yr. The low $\alpha$-ratios seen in Pal 12 must thus be ascribed a region with an star formation ongoing for a few gigayears, thus achieving full contributions from both Type I and Type II SN. Na is largely synthesized in massive stars, even more massive than those producing the bulk of the $\alpha$-elements, which subsequently explode as Type II SN. The extremely large deficiency of Na seen in Pal 12 thus is probably consistent with the low $\alpha$-element ratios and may not require an initial mass function with a deficit of very massive stars during the initial epochs of star formation.

The odd-even effect (see Fig. 1) is prominent in the region of the periodic table from $\mathrm{Na}$ to Co. Elements with even atomic numbers in this region of the periodic table all have their most abundant stable isotope containing even numbers of both protons and neutrons. Arnett (1971; see also Arnett 1996, p. 275) discussed the odd-even effect for production of these elements through explosive nucleosynthesis. He demonstrated that the amplitude of this effect depends on the neutron excess,

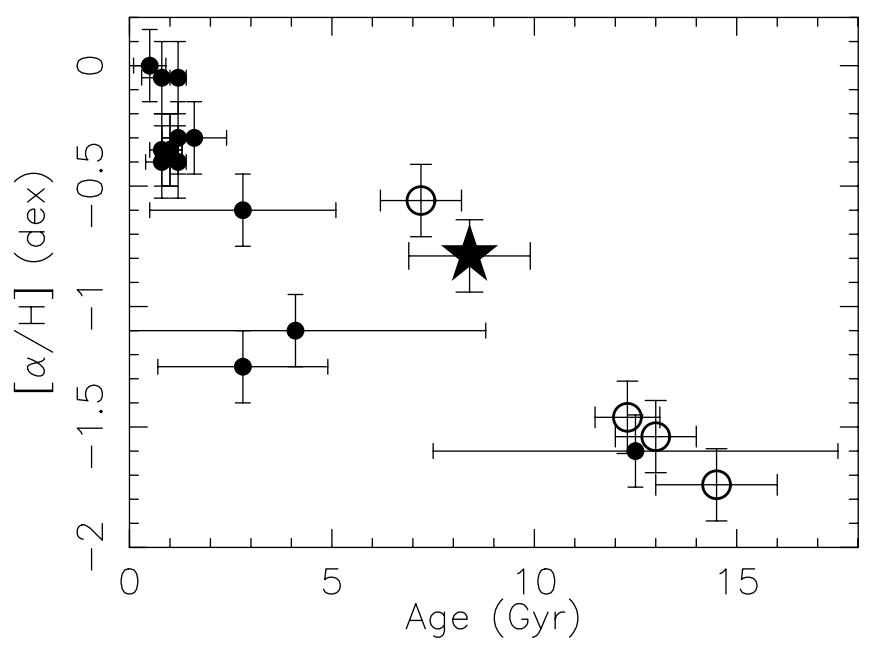

FIg. 5.-Plot of $[\alpha / \mathrm{H}]$ vs. age for stars in the Sgr dSph galaxy (data from Smecker-Hane \& McWilliam 2003; small filled circles), the four Galactic GCs believed to be associated with the Sgr stream (data largely from Layden \& Sarajedini 2000; large open circles), and for Pal 12 (large star). 
with larger amplitude for smaller neutron excesses which are characteristic of metal-poor material that has not been modified by $\mathrm{H}$ or He burning. It has long been clear that the oddeven effect is strongly enhanced among halo stars, and this effect is seen in Pal 12 as well.

While the presence of enhanced $\mathrm{Eu}$ requires a substantial $r$-process contribution in Pal 12, there may be contributions to its inventory of heavy elements from the $s$-process as well. The other key feature for the heavy elements in Pal 12 is the excess deficiency of $\mathrm{Y}$ and $\mathrm{Zr}$ compared with $\mathrm{Ba}$ and La. The $s$-process elements in stars with metallicities above $-1.5 \mathrm{dex}$ are believed to be formed mostly in asymptotic giant branch stars of intermediate mass. Nucleosynthesis in such conditions, reviewed by Busso, Gallino, \& Wasserburg (1999), with more recent calculations by Busso et al. (2001), is dependent on the ratio of free neutrons to seed nuclei, presumably Fe. A larger neutron-to-seed ratio will lead to increased production of the heavier $s$-process elements relative to the lighter ones, i.e., to those in the Ba peak versus those near $\mathrm{Sr}$, which is what is seen among the Pal 12 stars. In an extreme case, this leads to $s$-process production of detectable amounts of lead in very low metallicity stars (see, e.g., Cohen et al. 2003). Thus the anomalous ratios seen among the heavy elements in Pal 12 may be symptomatic of $s$-process nucleosynthesis at low metallicities.

Smecker-Hane \& McWilliam (2003) give a more detailed discussion of these ideas and how they apply to their sample of Sgr dSph stars spanning a wide range of metallicity and showing peculiarities that depend on their $[\mathrm{Fe} / \mathrm{H}]$.

\section{SUMMARY}

We present a detailed abundance analysis for 21 elements based on high-dispersion, high spectral resolution Keck/HIRES spectra for four members of the outer halo "young" Galactic GC Pal 12, which has an age of $\sim 8$ Gyr, $~ 30 \%$ younger than almost all GCs (Rosenberg et al. 1998), and is known to have a rather high metallicity for a GC in the outer halo (Brown et al. 1997). Since the discovery of the Sgr dSph galaxy, the Galactic GCs M54, Arp 2, Terzan 7, and Terzan 8 have been believed to be associated with this galaxy, which is currently being accreted by the Milky Way. Irwin (1999) recently suggested that Pal 12 is also a Galactic GC which has been tidally stripped from the Sgr galaxy.

All four stars in our sample in Pal 12 show identical abundance distributions with no credible indication of any star-to-star scatter. However, the abundance ratios of the Pal 12 stars are very peculiar. With $[\mathrm{Fe} / \mathrm{H}] \sim-0.8 \mathrm{dex}$, there is no detected enhancement of the $\alpha$-elements; the mean of [ $\mathrm{Si} / \mathrm{Fe}]$, $[\mathrm{Ca} / \mathrm{Fe}]$, and $[\mathrm{Ti} / \mathrm{Fe}]$ is $-0.07 \pm 0.05 \mathrm{dex}, \mathrm{O} / \mathrm{Fe}$ is also solar, while $\mathrm{Na}$ is very deficient. The distribution among the heavy elements shows anomalies as well, with a shift from the first $s$-process peak ( $\mathrm{Y}$ and $\mathrm{Zr}$ ) to the second peak ( $\mathrm{Ba}$ and $\mathrm{La}$ ). (Eu is highly enhanced, as is typical in Galactic GCs of similar metallicity.) We show that these abundance peculiarities are not seen among other stellar populations in the Galaxy, including almost all Galactic GCs and field stars in the disk, halo, and bulge of the Galaxy.

The abundance anomalies shown by the Pal 12 stars are, however, in good general agreement with the trends established by Smecker-Hane \& McWilliam (2003) and Bonifacio et al. (2000) for stars in the Sgr dSph galaxy, when evaluated at the $[\mathrm{Fe} / \mathrm{H}]$ of Pal 12. It is interesting to note that the trend of low/no $\alpha$-enhancement has been found in all high-metallicity stars in all the dSph satellites of the Galaxy studied to date. The abundance peculiarities exhibited by Pal 12 can in general be explained by the initiation of star formation in a metal-poor system and an extended period of star formation lasting at least a few gigayears.

Our abundance analysis of a sample of four stars in Pal 12 thus reinforces earlier suggestions that this GC originally was a cluster in the Sgr dSph galaxy that during the process of accretion of this galaxy by our own was tidally stripped from the Sgr galaxy to become part of the extended Sgr stream. Further searches for such anomalies are perhaps best concentrated among metal-rich outer halo Galactic GCs; in the metal-poor ones, the duration of the major epoch of star formation may have been too brief for significant differences to develop in star formation history and hence in abundance distributions among the GCs.

This paper is for Chris Sneden, who at his review talk in 2003 February at the Carnegie symposium on "The Origin and Evolution of the Elements" appealed for someone to take another look at Pal 12. The entire Keck/HIRES user communities owes a huge debt to Jerry Nelson, Gerry Smith, Steve Vogt, and many other people, who have worked to make the Keck Telescope and HIRES a reality and to operate and maintain the Keck Observatory. We are grateful to the W. M. Keck Foundation for the vision to fund the construction of the W. M. Keck Observatory. The author wishes to extend special thanks to those of Hawaiian ancestry on whose sacred mountain we are privileged to be guests. Without their generous hospitality, none of the observations presented herein would have been possible. We are grateful to the National Science Foundation for partial support under grant AST 02-05951 to J. G. C. We thank Jason Prochaska and Andy McWilliam for providing their tables of hyperfine structure in digital form, and Andy McWilliam and Tammy Smecker-Hane for providing their Sgr dSph abundances in digital form in advance of publication.

\section{REFERENCES}

Allende Prieto, C., Lambert, D. L., \& Asplund, M. 2001, ApJ, 556, L63

Armandroff, T. E., \& Da Costa, G. S. 1991, AJ, 101, 1329

Arnett, W. D. 1971, ApJ, 166, 153

Arnett, D. 1996, Supernovae and Nucleosynthesis: An Investigation of the History of Matter, from the Big Bang to the Present (Princeton: Princeton Univ. Press)

Bellazzini, M., Ibata, R., Ferraro, R., \& Testa, V. 2003, A\&A, 405, 577

Bonifacio, P., Hill, V., Molaro, P., Pasquini, L., Di Marcantonio, P., \& Santin, P. 2000, A\&A, 359, 663

Bonifacio, P., Sbordone, S., Marconi, G., Pasquini, L., \& Hill, V. 2003, A\&A, in press

Brown, J. A., Wallerstein, G., \& Gonzalez, G. 1999, AJ, 118, 1245

Brown, J. A., Wallerstein, G., \& Zucker, D. 1997, AJ, 114, 180
Burris, D. L., Pilachowski, C. A., Armandroff, T. E., Sneden, C., Cowan, J. J., \& Roe, H. 2000, ApJ, 544, 302

Busso, M., Gallino, R., Lambert, D. L., Travaglio, C., \& Smith, V. V. 2001, ApJ, 557, 802

Busso, M., Gallino, R., \& Wasserburg, G. J. 1999, ARA\&A, 37, 239

Carney, B. W., Wright, J. S., Sneden, C., Laird, J. B., Aguilar, L. A., \& Latham, D. W. 1997, AJ, 114, 363

Cohen, J. G., Behr, B. B., \& Briley, M. M. 2001, AJ, 122, 1420

Cohen, J. G., Christlieb, N., Qian, Y. Z., \& Wasserburg, G. J. 2003, ApJ, 588, 1082

Cohen, J. G., Frogel, J. A., \& Persson, S. E. 1978, ApJ, 222, 165

Den Hartog, E. A., Lawler, J. E., Sneden, C., \& Cowan J. J. 2003, ApJS, 148,543 
Dinescu, D. I., Majewski, S. R., Girard, T. M., \& Cudworth, K. M. 2000, AJ, 120,1892

Fuhr, J. R., Martin, G. A., \& Wiese, W. L. 1988, J. Phys. Chem. Ref. Data 17, Suppl. 4

Fulbright, J. P. 2002, AJ, 123, 404

Gratton, R. G., Carretta, E., Eriksson, K., \& Gustafsson, B. 1999, A\&A, 350,955

Gratton, R. G., \& Ortolani, S. 1988, A\&AS, 73, 137

Grevesse, N., \& Sauval, A. J. 1998, Space Sci. Rev., 85, 161

Gustafsson, B., Bell, R. A., Eriksson, K., \& Nordlund, Å. 1975, A\&A, 42, 407

Han, Z., \& Podsiadlowski, Ph. 2003, MNRAS, in press

Hannaford, P., Lowe, R. M., Grevesse, N., Biemont, E., \& Whaling, W. 1982, ApJ, 261, 736

Harris, W. E. 1996, AJ, 112, 1487

Harris, W. E., \& Canterna, R. 1980, ApJ, 239, 815

Houdashelt, M. L., Bell, R. A., \& Sweigart, A. V. 2000, AJ, 119, 1448

Ibata, R. A., Irwin, M. J., \& Gilmore, G. 1994, Nature, 370, 194

Ibata, R., Lewis, G. F., Irwin, M., Totten, E., \& Quinn, T. 2001, ApJ, 551, 294

Irwin, M. J. 1999, in IAU Symp. 192, The Stellar Content of Local Group Galaxies, ed. P. A.Whitelock \& R. D Cannon (San Francisco: ASP), 409

Ivans, I. I., Sneden, C., James, C. R., Preston, G. W., Fulbright, J. P., Hoflich, P. A., Carney, B. W., \& Wheeler, J. C. 2003, ApJ, 592, 906

Ivezić, Ž., et al. 2003, in Milky Way Surveys: The Structure and Evolution of Our Galaxy, ed. D. Clemens \& T. Brainerd (San Francisco: ASP), in press

Käppeler, F., Beer, H., \& Wisshak, K. 1989, Rep. Prog. Phys., 52, 945

Kurucz, R. L. 1993a, CD-ROM 13, ATLAS9 Stellar Atmosphere Programs and $2 \mathrm{~km} / \mathrm{s}$ Grid (Cambride: SAO)

Lawler, J. E., Bonvallet, G., \& Sneden, C. 2001a, ApJ, 556, 452

Lawler, J. E., Wickliffe, M. E., den Hartog, E. A., \& Sneden, C. 2001b, ApJ, 563,1075

Layden, A. C., \& Sarajedini, A. 2000, AJ, 119, 1760

Majewski, S. R., Skrutskie, M. F., Weinberg, M. D., \& Ostheimer, J. C. 2003, ApJ, 599, 1082

Martin, G. A., Fuhr, J. R., \& Wiese, W. L. 1988, J. Phys. Chem. Ref. Data 17, Suppl. 3

Martinez-Delgado, D., Zinn, R., Carrera, R., \& Gallart, C. 2002, ApJ, 573, L19

Mashonkina, L., \& Gehren, T. 2000, A\&A, 364, 249

Mashonkina, L., Gehren, T., \& Bikmaev, I. 1999, A\&A, 343, 519

McWilliam, A. 1997, ARA\&A, 35, 503 1998, AJ, 115, 1640

McWilliam, A., Rich, R. M., \& Smecker-Hane, T. A. 2003, ApJ, 592, L21 (erratum 593, L145)

Moore, C. E., Minnaert, M. G. J., \& Houtgast, J. 1966, The Solar Spectrum $2935 \AA$ to $8770 \AA$, National Bureau of Standards Monograph (Washington, D.C.: USGPO)

Nissen, P. E. 2004, in Origin and Evolution of the Elements, ed. A. McWilliam \& M. Rauch (Cambridge: Cambridge Univ. Press), in press

Nissen, P. E., \& Schuster, W. J. 1997, A\&A, 326, 751
Prochaska, J. X., Naumov, S. O., Carney, B. W., McWilliam, A., \& Wolfe, A. M. 2000, AJ, 120, 2513

Ramírez, S. V., \& Cohen, J. G. 2002, AJ, 123, 3277 2003, AJ, 125, 224

Ramírez, S. V., Cohen, J. G., Buss, J., \& Briley, M. M. 2001, AJ, 122, 1429

Reddy, B. E., Tomkin, J., Lambert, D. L., \& Allende-Prieto, C. 2003, MNRAS, 340,304

Rosenberg, A., Saviane, I., Pioto, G., \& Aparicio, A. 1999, AJ, 118, 2306

Rosenberg, A., Saviane, I., Piotto, G., \& Held, E. V. 1998, A\&A, 339, 61

Sbordone, L., Bonifacio, P., Marconi, G., \& Buonanno, R. 2003, Poster Pres. Joint Disc. 4, Astrophysical Impact of Abundance in Globular Clusters

Schlegel, D. J., Finkbeiner, D. P., \& Davis, M. 1998, ApJ, 500, 525

Shetrone, M. D., Bolte, M., \& Stetson, P. B. 1998, AJ, 115, 1888

Shetrone, M. D., Côté, P., \& Sargent, W. L. W. 2001, ApJ, 548, 592

Shetrone, M. D., Venn, K. A., Tolstoy, E., Primas, F., Hill, V., \& Kaufer, A. 2003, AJ, 125, 684

Shortridge, K. 1993, in ASP Conf. Ser. 52, Astronomical Data Analysis Software and Systems II, ed. R. J. Hannisch, R. J. V. Brissenden, \& J. Barnes (San Francisco: ASP), 219

Skrutskie, M. F., et al. 1997, in The Impact of Large Scale Near-IR Sky Surveys, ed. F. Garzon et al. (Dordrecht: Kluwer), 187

Smecker-Hane, T. A., \& McWilliam, A. 2003, ApJ, submitted (astro-ph/0205411)

Sneden, C. 1973, Ph.D. thesis, Univ. Texas, Austin

Stephens, A. 1999, AJ, 117, 1771

Stephens, A., \& Boesgaard, A. M. 2002, AJ, 123, 1647

Stetson, P. B., Vandenberg, D. A., Bolte, M., Hesser, J. E., \& Smith, G. H. 1989, AJ, 97, 1360

Takeda, Y., Zhoa, G., Takada-Hidai, M., Chen, Y.-Q., Saito, Y., \& Zhang, H.-W. 2003, Chinese J. Astron. Astrophys., 3, 316

Tautvaisiene, G., Wallerstein, G., Geisler, D., Gonzalez, G., \& Charbonnel, C. 2004, AJ, 127, 373

Tolstoy, E., Venn, K. A., Shetrone, M., Primas, F., Hill, V., Kaufer, A., \& Szeifert, T. 2003, AJ, 125, 707

Vogt, S. E., et al. 1994, Proc. SPIE, 2198, 362

Wallace, L., Hinkle, K., \& Livingston, W. C. 1998, An Atlas of the Spectrum of the Solar Photosphere from 13,500 to $28,000 \mathrm{~cm}^{-1}$ (3570 to $7405 \AA$ ) (NSO Tech. Rep. 98-001) (Tucson: Natl. Sol. Obs.)

Wallerstein, G., Gonzalez, G., \& Geisler, D. 2002, in IAU Symp. 207, Extragalactic Star Clusters, ed. D. Geisler, E. Grebel, \& D. Minitti (San Francisco: ASP), 171

Wiese, W. L., Fuhr, J. R., \& Deters, T. M. 1996, J. Phys. Chem. Ref. Data, Monograph 7

Wiese, W. L., Smith, M. W., \& Miles, B. M. 1969, Atomic Transition Probabilities, Vol. 2: Sodium through Calcium (NSRDS-NBS 22) (Washington, D.C.: Dept. Comm., Natl. Bur. Stand.)

Wheeler, J. C., Sneden, C., \& Truran, J. W. 1989, ARA\&A, 27, 279

Yi, S., Demarque, P., Kim, Y.-C., Lee, Y.-W., Ree, C., Lejeune, Th., \& Barnes, S. 2001, ApJS, 136, 417 